Secondary school students educate peers on good oral health

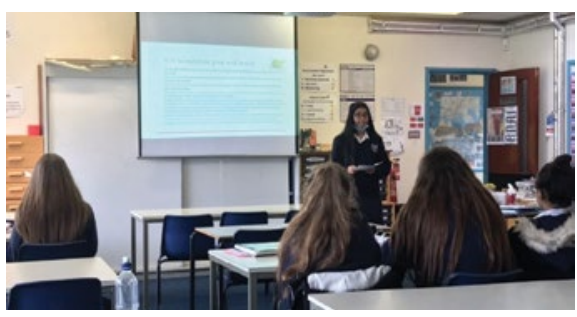

One of the students delivering the presentation. Due to social distancing the others had to take part using Zoom

A group of year 13 students in the West Midlands teamed up with a local dentist to deliver a presentation on 'How nutrition affects oral health' to their year groups.

Fatima Malik wrote to the $B D J$ on behalf of the group, which also included Fatima Imran, Kulsoom Sakha, Sanaz Mirza and Rubab Mahmood.

Fatima said: 'We are passionate about spreading awareness about preventative dentistry as we realise it is often neglected by many people, especially those our age. This is because a lot of them are unaware and do not realise the importance of taking care of your teeth and the impact a bad diet, especially sugary foods, can have on your oral health'

The group teamed up with Dr Uzair Janjua, a dentist who mentors young students who aspire to be dentists. In the presentation, the students gave tips on how to maintain good oral hygiene and explained what foods or drinks fellow students should avoid, as well as warning them about the potential negative consequences of bad oral health.

Fatima said: 'After giving the presentation a lot of our classmates and even teachers were telling us about how they were unaware of how much of an impact a bad diet can have on their oral health and how bad oral health is linked to many severe health problems. Many of them were thanking us, saying how they learnt a lot and will try their best to use our tips to maintain better oral hygiene'.

All of the year 13 students involved in the presentation are applying to dental school this year. Fatima was inspired through witnessing her father's journey through periodontal disease and seeing the negative impacts smoking and not maintaining good oral hygiene had on his teeth. The students hope to go on raising awareness of the importance of maintaining good oral health.

\title{
FDS calls for dentistry 'to be kept open' throughout pandemic
}

As three in four dentists (74\%) say they

will be unable to provide a full service

this year due to COVID-19, the Faculty of

Dental Surgery (FDS) at the Royal College of Surgeons of England has called on the government to provide every possible support to dentistry.

The FDS surveyed more than 450 dentists to understand what barriers remain to resuming services, which were cancelled in the wider efforts to prevent the spread of COVID-19.

As dental services began to resume over the summer, nearly all dentists surveyed (96\%) said they were providing a service, but not the 'full service' that they would have provided before the pandemic. Private practice respondents were considerably more likely to have provided vitally important 'aerosol generating procedures', most commonly associated with the use of a normal dental drill, with $83 \%$ having done so. In NHS hospital practice, this fell to $62 \%$, and fell further to $40 \%$ in NHS general dental practice.

When looking ahead, a third (34\%) of respondents did not know when they would fully resume services, with a further $36 \%$ saying they did not expect to resume full pre-pandemic services until 2021. Overall, three in four (74\%) said they would be unable to provide a full service this year or don't know when they will be able to do so.

Comments revealed that time taken between each patient to comply with new infection control protocols brought in because of COVID-19 was slowing down how many patients could be seen. Dentists are seeing far fewer patients per 'session' (half day) than prior to the pandemic. Where $46 \%$ of dentists were able to see more than ten patients each morning or afternoon before COVID-19, only $7 \%$ can say the same now.

Only half (48\%) of NHS general practice dentists said they had an adequate supply of PPE to enable them to do their job safely. Dentists working in hospitals, however, were much more confident, with
$80 \%$ agreeing they had adequate supplies.

Leading dental surgeons from the Faculty of Dental Surgery on 2 October said that services 'should not be switched off again' in a second wave, as dentistry is an essential part of healthcare. In a new report, A resumption of dental services? Dental surgeons' experiences of delivering care since 8 June 2020 (https:// bit.ly/3lBCnff), the Faculty calls on the UK government to:

1. Keep dentistry open throughout the remaining 'stages' of the COVID19 pandemic, using PPE and infection prevention measures to mitigate risks and keep patients safe

2. Ensure adequate PPE across regions and settings. Dental surgeons across specialties remain reliant on PPE to continue to provide dental care. NHS general practice dentists need parity of access with NHS hospital practices, to ensure patients have access to safe treatment

3. Tackle the barriers to full resumption of dental care, particularly 'fallow time', by promoting and monitoring the use of ventilation systems to increase the number of 'air changes per hour' in dental settings.

The Dean of the Faculty, Mr Matthew Garrett said: 'We need urgent action in particular to help NHS general practice surgeries resume routine procedures like fillings and crown repair. These help the long-term good oral health of our patients and help prevent unnecessary dental extractions.

'It is also crucial that dentists are able to see more patients each day, getting back to pre-pandemic levels as soon as possible. This can be achieved with better ventilation systems in surgeries, and with an adequate supply of PPE.

'As with the rest of society, dentistry is going to have to "live with" COVID for the foreseeable future. We need every support possible to open services up again and keep them running no matter what.' 